Meta

Journal des traducteurs

Translators' Journal

\title{
La voix passive dans les textes médicaux et para-médicaux
}

\section{Maurice Rouleau}

Volume 38, numéro 3, septembre 1993

URI : https://id.erudit.org/iderudit/004002ar

DOI : https://doi.org/10.7202/004002ar

Aller au sommaire du numéro

\section{Éditeur(s)}

Les Presses de l'Université de Montréal

ISSN

0026-0452 (imprimé)

1492-1421 (numérique)

Découvrir la revue

Citer cet article

Rouleau, M. (1993). La voix passive dans les textes médicaux et para-médicaux. Meta, 38(3), 440-448. https://doi.org/10.7202/004002ar

\section{Résumé de l'article}

Dans les textes médicaux et para-médicaux, anglais ou français, les formes passives ne représentent que $33 \%$ ou $42 \%$ de toutes les formes verbales relevées, selon que les participes passés utilisés seuls sont considérés respectivement comme des adjectifs ou comme des formes verbales. Ce pourcentage semble cependant être influencé par la nature du texte. En français médical ou para-médical, le nombre déformes verbales actives construites avec le sujet " on » [on + verbe actif] ou déformes verbales pronominales est faible. La nature du texte semble influer sur la fréquence du " on " mais non sur celle des verbes pronominaux. Toutefois, traduire un verbe passif d'un texte médical ou para-médical par [on + v. actif] ou par un verbe pronominal fait peut-être " français " mais ne fait ni « scientifique " ni " médical ". 


\title{
LA VOIX PASSIVE DANS LES TEXTES MÉDICAUX ET PARA-MÉDICAUX
}

MAURICE RoUlEAU

Université du Québec à Trois-Rivières, Trois-Rivières, Canada

\begin{abstract}
Résumé
Dans les textes médicaux et para-médicaux, anglais ou français, les formes passives ne représentent que $33 \%$ ou $42 \%$ de toutes les formes verbales relevées, selon que les participes passés utilisés seuls sont considérés respectivement comme des adjectifs ou comme des formes verbales. Ce pourcentage semble cependant être influencé par la nature du texte. En français médical ou para-médical, le nombre de formes verbales actives construites avec le sujet "on" [on + verbe actif] ou de formes verbales pronominales est faible. La nature du texte semble influer sur la fréquence du «on» mais non sur celle des verbes pronominaux. Toutefois, traduire un verbe passif d'un texte médical ou para-médical par [on + v. actif] ou par un verbe pronominal fait peut-être "français» mais ne fait ni «scientifique» ni «médical».
\end{abstract}

\section{INTRODUCTION}

Traduire consiste essentiellement à réexprimer efficacement dans une autre langue le message que renferme le texte de départ. Le produit d'une telle opération doit, pour être jugé correct, répondre à divers critères de qualité :

1) transmettre exactement le message contenu dans le texte de départ;

2) observer les normes grammaticales actuelles de la langue d'arrivée;

3) être idiomatique;

4) être dans le même ton que l'original (équivalence stylistique);

5) être pleinement intelligible pour le lecteur qui appartient à une autre culture (équivalence culturelle) (Darbelnet 1970).

Exigées pour toutes les traductions, ces cinq qualités n'ont cependant pas la même importance pour tous les genres de textes. Il est rare, par exemple, qu'un traducteur spécialisé dans le domaine médical ou scientifique doive faire face à des problèmes d'ordre stylistique. Les textes qu'il aura à traduire se caractérisent essentiellement par leur fonction informative; les effets de style sont plutôt le lot du traducteur littéraire. L'un comme l'autre par contre devront rendre leur texte dans une langue idiomatique, une langue qui ne permet pas de savoir qu'il s'agit d'une traduction.

Pour se faire, le traducteur se doit de connaître les caractéristiques des langues dans lesquelles il travaille. On lui a enseigné, par exemple - à tort ou à raison - que le français préfère les formes nominales et l'anglais les constructions verbales; que, contrairement à l'anglais, le français antépose généralement les compléments circonstanciels, etc. Bien que ces caractéristiques soient souvent énoncées, il est rare que le type de discours auxquelles elles s'appliquent soit précisé.

Prenons le cas du passif. Qui n'a jamais entendu dire, par exemple, que l'anglais adore le passif, mais que le français l'abhorre? ou encore, à l'instar de Koessler (1975: 32), que: «La préférence que l'anglais donne au passif dans l'énonciation de sa pensée peut être considérée comme idiomatique.» Sous cette forme, l'énoncé se rapproche étrangement 
d'une proposition universelle. Et son corollaire (l'emploi du passif n'est pas idiomatique en français) amène le traducteur francophone à ne pas utiliser un passif pour traduire un passif anglais.

Le point de vue de J.-P. Attal (1987: 791-792) se rapproche de celui de M. Koessler, mais il est plus nuancé.

Bien que, dans l'ensemble, la construction active soit statistiquement plus employée en anglais que la construction passive, il y a de nombreuses exceptions particulières. La forme passive est préférée par exemple dans le style documentaire descriptif des articles de journaux ou dans la langue scientifique. Par contre, c'est la forme active qui l'emporte en littérature où l'on insiste plus sur les personnes actives.

Si cela est vrai, ce qui est idiomatique en langue générale ne le serait donc pas nécessairement en langue de spécialité. S'il en est ainsi en anglais, qu'en est-il en français? Cette question rejoint d'ailleurs une préoccupation des étudiants en version médicale ou scientifique qui se demandent comment traduire les passifs anglais. Faut-il, comme on l'enseigne, employer un actif avec un sujet indéterminé («on») (ex.: on a observé ces phénomènes), ou déterminé (ex.: le chercheur a observé ces phénomènes), ou encore recourir à la voix pronominale (ex.: ces phénomènes s'observent) pour laquelle le français aurait une affinité (Vinay et Darbelnet 1977: 134) ou tout simplement maintenir la voix passive (ex.: ces phénomènes ont été observés)?

Cet article se propose d'explorer sommairement la façon qu'a de s'exprimer par ecrit le scientifique francophone qui œuvre dans les domaines médical et para-médical. Utilise-t-il de préférence la tournure active? Recourt-il souvent à la voix pronominale ou privilégie-t-il plutôt la construction passive? Les résultats de cette analyse devraient permettre à celui qui veut traduire un texte médical ou para-médical d'accorder sa façon d'écrire avec la façon de dire des spécialistes du domaine, autrement dit de produire un texte idiomatique.

\section{MATÉRIEL ET MÉTHODES}

Les textes spécialisés retenus pour cette étude font partie d'un seul domaine de connaissances, celui des sciences de la santé (Tableau 1). Comme ce domaine n'est pas monolithique, il est possible que le type de discours utilisé ne soit pas unique. Ainsi, pour éviter que le type de discours interfère dans la comparaison, chaque article français représentant un type particulier a été jumelé à un article de même type écrit en anglais. Le choix des sujets des 10 articles a été déterminé par le hasard. La seule contrainte a été la nécessité de trouver deux articles, un en français et un en anglais, traitant idéalement du même sujet.

Les types de discours retenus sont ceux que le traducteur médical est susceptible de rencontrer dans l'exercice de ses fonctions. Au nombre de cinq, ces types peuvent être groupés en deux catégories : les textes dits «scientifiques» et les textes dits «généraux».

Dans les articles dits «scientifiques», articles où les auteurs rapportent les résultats de leurs travaux expérimentaux, les sujets traités ne sont pas de même nature. Dans les articles 1 et 2 , il s'agit d'interventions chirurgicales; dans les articles 3 et 4 , d'expériences de laboratoire dont les variables étudiées peuvent être modifiées par le chercheur; dans les articles 5 et 6 , d'observations en histopathologie humaine, domaine où les données peuvent être quantifiées mais où les variables ne peuvent être modifiées par l'expérimentateur. Ces trois types se différencient donc par le degré d'intervention du chercheur dans l'obtention des résultats. 


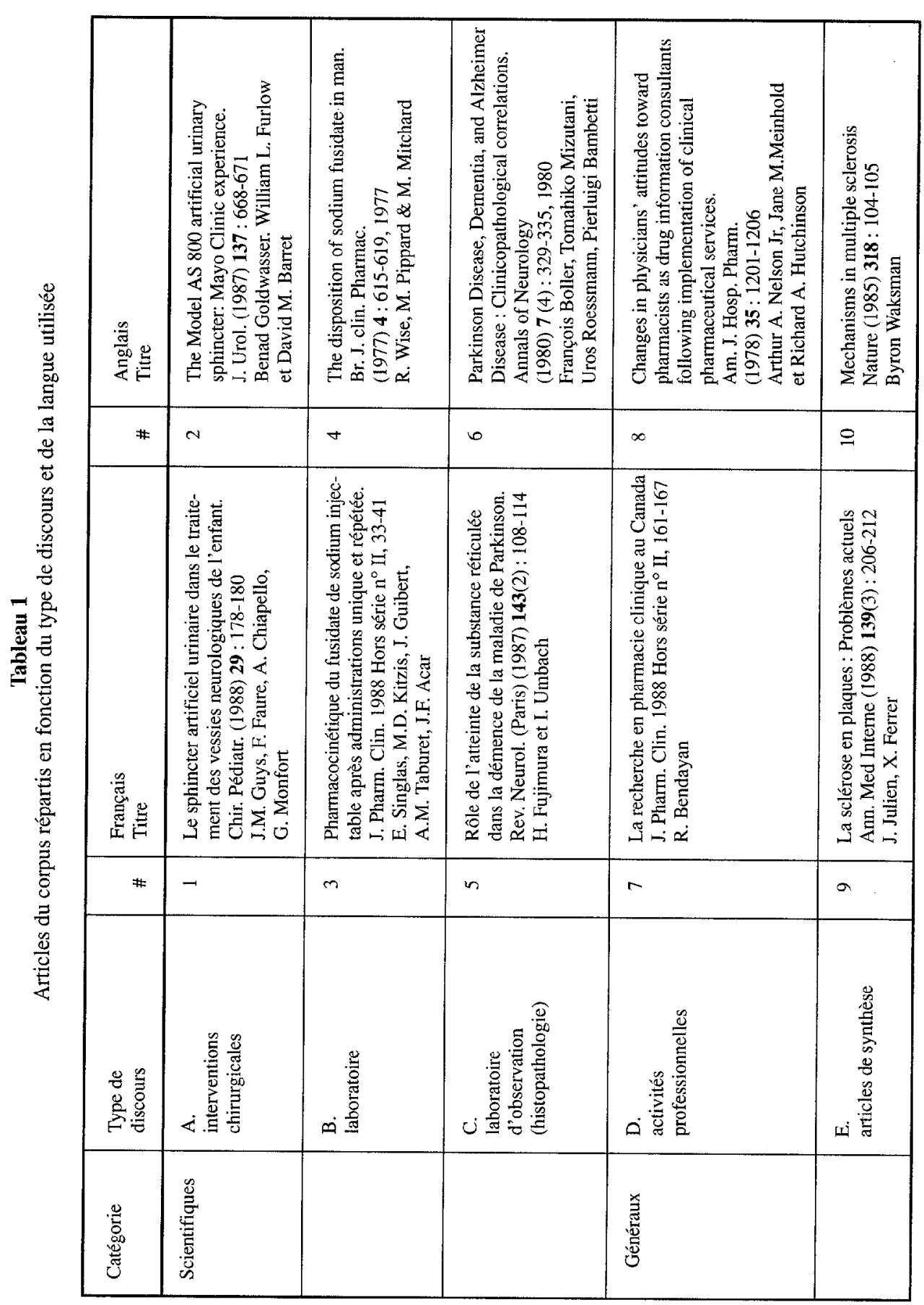


Quant aux articles dits «généraux», ils présentent le point de vue du spécialiste sur un sujet qui ne se rapporte pas directement à sa propre activité de recherche. Les articles choisis traitent de la vie professionnelle (articles 7 et 8 ) ou font la synthèse d'un sujet de recherche (articles 9 et 10). Ce dernier groupe d'articles constitue ce qui pourrait être appelé le discours généraliste du spécialiste.

Les résumés des articles écrits dans une langue autre que la langue originale de l'article n'ont pas été pris en considération dans cette étude. Ayant probablement été rédigés par des personnes dont la langue maternelle n'était pas la langue du résumé, ils ne présentent aucun intérêt pour qui veut savoir comment s'exprime dans sa propre langue un scientifique œuvrant dans le domaine des sciences de la santé.

Toutes les formes verbales conjuguées ont été retenues à l'exception des verbes modulateurs. Les seuls verbes à l'infinitif à avoir été pris en considération sont ceux qui étaient introduits par un modulateur; ils ont été classés actifs ou passifs selon le sens (could indicate $=$ actif $;$ should not be detected $=$ passif $)$.

L'étude a porté sur plus de 2000 formes verbales réparties presque également entre les deux langues: 998 en français et 1053 en anglais.

\section{RÉSULTATS}

\section{Fréquences globales des formes verbales ayant valeur de passif}

Que le texte soit écrit en français ou en anglais, le pourcentage moyen de formes verbales ayant valeur de passif dans les 5 types de discours analysés est de $42 \%$ (Tableau 2). Toutes les valeurs obtenues ont servi au calcul des moyennes, car rien statistiquement ne justifie que l'une d'entre elles soit exclue. Les valeurs individuelles varient légèrement, mais elles sont toutes inférieures à $50 \%$, à une exception près (les articles 2 et 3 ). L'influence de la valeur particulière de l'article 2 sur le pourcentage moyen en français se trouve annulée par la présence de la même particularité dans le texte anglais (article 3).

Tableau 2

Répartitions globales des formes ayant valeur de passif

\begin{tabular}{|cccccccc|}
\hline & \multicolumn{3}{c}{ FRANÇAIS } & \multicolumn{5}{c|}{ ANGLAIS } \\
\hline$\#$ & $\mathrm{~N}$ & $\begin{array}{c}\text { Passif } \\
(\%)\end{array}$ & $\begin{array}{c}\text { Autres } \\
(\%)\end{array}$ & $\#$ & $\mathrm{~N}$ & $\begin{array}{c}\text { Passif } \\
(\%)\end{array}$ & $\begin{array}{c}\text { Autres } \\
(\%)\end{array}$ \\
\hline 1. & 91 & 48 & 52 & 2. & 215 & 34 & 66 \\
3. & 127 & 60 & 40 & 4. & 191 & 53 & 47 \\
5. & 139 & 43 & 57 & 6. & 260 & 39 & 61 \\
7. & 161 & 27 & 73 & 8. & 261 & 39 & 61 \\
9. & 480 & 31 & 69 & 10. & 126 & 47 & 53 \\
\hline$\sum$ & 998 & & & & 1053 & & \\
$\%$ & 42 & 58 & & & 42 & 58 \\
\hline
\end{tabular}

Si les résultats du Tableau 2 sont analysés en fonction des catégories de discours (textes dits «scientifiques» vs textes «généraux»), une particularité semble se dégager. En français, les textes «scientifiques» contiennent un pourcentage plus fort de formes 
passives $(48 \%, 60 \%$ et $43 \%)$ que les textes «généraux» $(27 \%$ et $31 \%)$. Ces résultats contrastent avec ceux des textes anglais où la catégorie de discours n'a aucune influence; les pourcentages moyens des deux mêmes groupes sont respectivement de $42 \%$ et de $43 \%$.

Les formes ayant valeur de passif qui ont été prises en considération dans cette analyse englobent à la fois les participes passés des temps composés avec être et ceux qui ont valeur d'un simple qualificatif épithète ou attribut (Grevisse 1980: \#1898-1899). Que ces derniers soient ou non pris en considération dans les calculs, la conclusion générale demeure la même (comparer le Tableau 2 au Tableau 3) : le pourcentage des formes passives est toujours inférieur à $50 \%$, aussi bien en français qu'en anglais. Les formes passives ne sont donc pas dominantes.

Tableau 3

Répartition des formes verbales à l'exclusion des participes passés considérés comme adjectifs ou attributs

\begin{tabular}{|lccccc|}
\hline \multicolumn{3}{|c}{ FRANÇAIS } & \multicolumn{3}{c|}{ ANGLAIS } \\
\hline$\#$ & $\begin{array}{c}\text { Passif } \\
(\%)\end{array}$ & $\begin{array}{c}\text { Autres } \\
(\%)\end{array}$ & $\#$ & $\begin{array}{c}\text { Passif } \\
(\%)\end{array}$ & $\begin{array}{c}\text { Autres } \\
(\%)\end{array}$ \\
\hline 1. & 40 & 60 & 2. & 25 & 75 \\
3. & 44 & 56 & 4. & 44 & 56 \\
7. & 13 & 87 & 8. & 28 & 72 \\
\hline$\%$ & 32 & 68 & & 32 & 68 \\
moyen & & & & & \\
\hline
\end{tabular}

\section{Répartition des formes qui n'ont pas valeur de passif}

Pour connaître la répartition des formes qui n'ont pas valeur de passif, six des dix articles du corpus ont été dépouillés. Le verbe «être» constitue près du tiers de ces formes (Tableau 4). Ce pourcentage moyen est du même ordre de grandeur, que les textes soient écrits en français ou en anglais. Cependant, parmi les textes français, il semble exister une particularité: l'article 7, seul texte d'ordre général utilisé dans cette sous-analyse, contient beaucoup moins de verbe «être» que les deux autres textes dits «scientifiques». Ce phénomène ne s'observe cependant pas en anglais.

Tableau 4

Répartition des formes verbales qui n'ont pas valeur de passif

\begin{tabular}{|lccccccccc|}
\hline \multicolumn{1}{|c|}{ FRANÇAIS } \\
\hline$\#$ & $\mathrm{~N}$ & $\begin{array}{c}\text { ÊTRE } \\
(\%)\end{array}$ & $\begin{array}{c}\text { AVOIR } \\
(\%)\end{array}$ & $\begin{array}{c}\text { AUTRES } \\
(\%)\end{array}$ & $\#$ & $\begin{array}{c}\mathrm{N} \\
(\%)\end{array}$ & $\begin{array}{c}\text { ETRE } \\
(\%)\end{array}$ & $\begin{array}{c}\text { AVOIR } \\
(\%)\end{array}$ & AUTRES \\
\hline 1. & 47 & 38 & 0 & 62 & 2. & 140 & 31 & 6 & 63 \\
3. & 51 & 43 & 0 & 57 & 4. & 89 & 34 & 2 & 63 \\
7. & 117 & 11 & 2 & 87 & 8. & 160 & 30 & 4 & 66 \\
\hline$\%$ moyen & 31 & 0,7 & 69 & & & 32 & 4 & 64 \\
\hline
\end{tabular}


Quant au verbe «avoir», il se rencontre rarement dans les textes anglais. En français, il est encore moins présent et, dans les textes «scientifiques», il est absent; il ne se trouve que dans le texte «général».

\section{Manières de traduire un passif anglais.}

Même si le traducteur sait qu'il existe trois façons de rendre en français un verbe passif anglais («on» + v. actif, v. pronominal ou v. passif), cela ne l'aide pas à faire son choix. Pour pouvoir rendre un texte idiomatique, le traducteur doit connaître la façon de dire du scientifique cuvrant dans le domaine des sciences de la santé et rédigeant ses articles dans sa langue maternelle. Pour documenter cette façon de dire, nous avons étudié la fréquence d'utilisation de ces différentes tournures dans les cinq textes français du corpus (Tableau 5).

Tableau 5

Fréquence dans les textes français des façons possibles de traduire un passif anglais

\begin{tabular}{|lccccc|}
\hline Catégorie & $\#$ & $\mathrm{~N}$ & $\begin{array}{c}\text { Passif } \\
(\%)\end{array}$ & $\begin{array}{c}\text { 《ON» } \\
(\%)\end{array}$ & $\begin{array}{c}\text { «Voix pronominale» } \\
(\%)\end{array}$ \\
\hline SCIENTIFIQUES & & & & & \\
& 1. & 45 & 98 & 0 & 2 \\
& 3. & 82 & 93 & 2 & 7 \\
\hline GÉNÉRAUX & 5. & 68 & 88 & 4 & 4 \\
& 7. & 50 & 88 & 8 & 8 \\
\hline$\%$ moyen & 9. & 173 & 86 & 9 & 5 \\
\hline
\end{tabular}

Parmi les trois tournures possibles, la plus utilisée est le passif ; elle représente en moyenne plus de $90 \%$. Le reste est partagé également entre les deux autres tournures. La fréquence moyenne d'utilisation de la voix pronominale $(5 \%)$ ne semble pas être influencée par la catégorie de discours. La situation est toutefois différente dans le cas du [on $+\mathrm{v}$. actif]. Sa fréquence, bien que faible, semble présenter une particularité : le pourcentage moyen de fréquence dans les textes dits «scientifiques» est de $2 \%(0 \%, 2 \%$ et $4 \%$ ) alors que dans les textes «généraux» il est de $8,5 \%(8 \%$ et $9 \%)$.

\section{DISCUSSION}

Les conclusions de cette étude ne sont pas conformes aux idées couramment véhiculées et mises en pratique en ce qui concerne la traduction des passifs anglais. Quand il traduit des textes médicaux ou para-médicaux, le traducteur, en formation ou en excrcice, a l'habitude de rendre les passifs anglais par [on $+v$. actif]. Ses textes en contiennent d'ailleurs tellement que cette surabondance trahit le fait qu'il s'agit d'un texte traduit.

L'analyse des articles médicaux et para-médicaux du corpus révèle que l'anglais ne privilégie pas le passif plus que le français: il y a autant de formes passives en anglais qu'en français (Tableau 2). Dire, comme Koessler, qu'en anglais l'utilisation du passif est 
idiomatique, ne serait donc pas vrai de toutes les catégories de discours. Cette analyse révèle également que, dans les textes anglais étudiés, la forme passive n'est pas la forme dominante: elle ne représente que $42 \%$ des formes verbales (Tableau 2). Le seul texte anglais qui réponde à la description qu'Attal fait des textes scientifiques est l'article 4 . C'est le seul qui présente statistiquement plus de formes passives. Cela n'est cependant vrai qu'à la condition que toutes les formes ayant valeur de passif soient prises en considération. En effet, si les participes passés utilisés seuls sont assimilés à des adjectifs, le pourcentage de passifs chute sous les $50 \%$ (Tableau 3). Cet article constitue un type bien défini de discours; il décrit des expériences de laboratoire dont les variables sont modifiables par l'expérimentateur. Cette particularité du discours mériterait d'être étudiée plus à fond, car elle s'observe également en français. Il s'agit peut-être d'un caractère d'espèce plutôt que d'un caractère de langue. Ce parallélisme entre les résultats obtenus en anglais et en français vient confirmer la justesse de notre appréhension que le discours ne soit pas monolithique; 1'utilisation de paires d'articles (voir Matériel et Méthodes) était donc justifiée. Si un seul article de ce type avait été inclus dans l'analyse, les conclusions générales auraient été biaisées.

Les valeurs moyennes de $42 \%$ (Tableau 2) n'ont été établies qu'en fonction d'une seule variable: la langue dans laquelle les articles sont écrits. Si, en plus, l'analyse tient compte des deux catégories de discours (textes dits «scientifiques» et textes «généraux»), il ressort que les textes «scientifiques» français (articles 1, 3 et 5) contiennent un plus fort pourcentage de formes passives $(48 \%, 60 \%$ et $43 \%$ ) que les textes «généraux» ( $27 \%$ et $31 \%$ ). En anglais, ce phénomène ne s'observe pas; les pourcentages pour ces deux catégories sont respectivement de $42 \%$ et de $43 \%$. Ces dernières valeurs ne sont pas en accord avec l'affirmation d'Attal qui veut qu'un texte scientifique contienne plus de passifs qu'un texte général. Cette discordance s'explique peut-être par le fait que l'auteur du texte général dont parle Attal n'est pas un scientifique qui traite d'un sujet autre que ses propres recherches, comme cela est le cas dans la présente étude. Il faut reconnaître qu'ici le discours généraliste du spécialiste anglophone est modelé sur son discours «scientifique» (ou l'inverse) et que cette similitude ne s'observe pas en français.

Il est généralement admis que le français fait un plus grand usage des verbes actifs que des verbes passifs. Les résultats des Tableaux 2 et 3 viennent corroborer cette idée. Cependant, il y aurait lieu d'examiner plus attentivement si cette caractéristique ne présente pas des particularités en fonction du type de discours comme le suggèrent les données du Tableau 3. En effet, les formes passives sont les moins fréquentes dans les trois cas étudiés, mais, dans le texte dit «général», le pourcentage est beaucoup moins élevé (13\% comparativement à $42 \%$ dans les textes dits «scientifiques»). Ce plus fort pourcentage s'explique peut-être par le fait que la tournure passive sert doublement l'auteur d'un texte scientifique. Son message doit être chosocentrique (Bédard 1986: 156) et impersonnel. Chosocentrique parce que tout son discours est centré sur la chose qu'il a étudiée et que, pour que l'accent soit mis sur la chose, cette dernière doit être sujet de la phrase. Impersonnel parce que l'auteur, en tant qu'agent, n'a rien à voir avec la nature même du phénomène décrit. En sciences, médicales ou autres, un phénomène n'est vrai que si, toutes conditions étant identiques, il peut être reproduit par n'importe qui. Il importe donc peu que la chose soit mise en évidence par un agent particulier. C'est sans doute ce qui explique la présence, dans ce genre de texte, d'un grand nombre de passifs inachevés (un passif sans complément d'agent).

L'impersonnalité se rend aussi par l'utilisation de tournures impersonnelles souvent construites avec le verbe "être», par exemple «il est évident, il est à remarquer, il est raisonnable d'envisager, etc.». Le pourcentage de verbe «être»dans les articles dits 
«scientifiques» est d'environ $40 \%$ alors qu'il n'est que de $11 \%$ dans l'article «général» (voir Tableau 4). Ce faible pourcentage s'explique peut-être par un besoin d'impersonnalité moins grand dans ce genre de texte. Par contre, ce phénomène ne s'observe pas en anglais, ce qui peut porter à conclure que le recours au passif est typique de l'anglais. Le rôle de l'auteur dans l'obtention des résultats (composante de l'impersonnalité du rapport) mériterait d'être étudié plus en détail. Par exemple, le fait que l'article 1 soit le seul à contenir le pronom personnel «nous» s'explique peut-être par le type de discours. Une comparaison des trois types de discours «scientifiques» (interventions chirurgicales, sciences exactes, sciences d'observation) pourrait être révélatrice à cet égard.

Se fondant sur l'idée véhiculée selon laquelle le français utilise plus d'actifs que de passifs (l'anglais en fait pourtant autant), le traducteur médical est amené, pour rendre les passifs anglais, à ne pas recourir à un passif. De plus, comme il a été prémuni contre l'animisme, il hésite à utiliser la voix pronominale. Il ne lui reste donc plus qu'à recourir à [on + v. actif], ce qu'il fait d'une manière presque abusive.

Pour être à la hauteur de sa tâche, le traducteur doit produire un texte idiomatique. Il lui faut, pour ce faire, savoir si les deux tournures, [on $+v$. actif] et voix pronominale, font partie des moyens stylistiques qu'utilise le scientifique francophone. Les résultats présentés au Tableau 5 sont révélateurs. L'utilisation de ces 2 moyens ( $5 \%$ dans chaque cas) est faible. Il convient cependant de remarquer la différence de fréquence du [on $+\mathrm{v}$. actif] selon le type de discours $(1,3,5$ vs 7,9$)$. Dans les textes dits «scientifiques», sa fréquence est faible $(0 \%, 2 \%$ et $4 \%)$, alors que, dans les textes dits «généraux», sa fréquence augmente $(8 \%$ et $9 \%$ ). Cette différence ne s'observe cependant pas en ce qui concerne les pronominaux.

Cette étude ne concerne en fait que le discours actuel. Les textes utilisés ont été écrits au cours des 15 dernières années. Il est donc possible que la façon d'écrire relevée dans ces articles reflète la prédominance actuelle de l'anglais comme langue de communication en sciences, en technique et même en médical (il suffit de se rappeler la célèbre affaire des Annales de l'Institut Pasteur). Pour vérifier cette possibilité, il suffirait de comparer ces textes à des articles écrits au cours de la première moitié du $\mathrm{XX}^{\mathrm{e}}$ siècle, alors que l'anglais n'avait pas le rôle prédominant qu'on lui reconnaît aujourd'hui comme moyen de communication.

Actuellement, un traducteur face à un texte médical ou para-médical ne devrait pas craindre d'utiliser des passifs. Il pourra toujours, s'il le désire, traduire un passif par [on + v. actif]. Il doit cependant savoir que, ce faisant, il recourt à une tournure très peu utilisée par le chercheur en sciences de la santé pour décrire les résultats de ses recherches. Par contre, quand ce même chercheur tient un discours plus général, il utilise un peu plus le «on», sans cependant en abuser. Quant à la tournure pronominale, sa fréquence est faible tant dans les textes dits «scientifiques» que dans les textes dits «généraux». En conclusion, l'utilisation du [on $+v$. actif] dans une traduction médicale ou para-médicale fait peut-être français, mais elle ne fait pas scientifique au sens large du terme. 


\section{RÉFÉRENCES}

ATTAL, J.-Pierre (1987) : Grammaire et usage de l'anglais, Paris-Gembloux, Éditions Duculot.

BÉDARD, Claude (1986): La traduction technique. Principes et pratique, Montréal, Linguatech.

DARBELNET, Jean (1970) : «Traduction littérale ou traduction libre ?», Meta, 15-2: 89, cité par P.A. Horguelin (1985) : Pratique de la révision, $2^{e}$ édition, Montréal, Linguatech, p. 14

GREVISSE, Maurice (1980): Le bon usage, 11 édition, Paris-Gembloux et Montréal, Duculot, ERPI.

KOCOUREK, Rostislav (1982) : La langue française de la technique et de la science, Wiesbaden, Brandsteller Verlag.

KOESSLER, M. (1975): Les faux amis des vocabulaires anglais et américain, Paris, Librairie Vuibert.

VINAY J.-P. et J. DARBELNET (1977) : Stylistique comparée du français et de l'anglais, Montréal, Beauchemin.

\section{REMERCIEMENTS}

L'auteur tient à remercier Mme Alexandra Thalès pour le dépouillement de quelques articles, de même que Mme Louise LeBlanc et M. Robert Dubuc pour leurs suggestions fort judicieuses. 